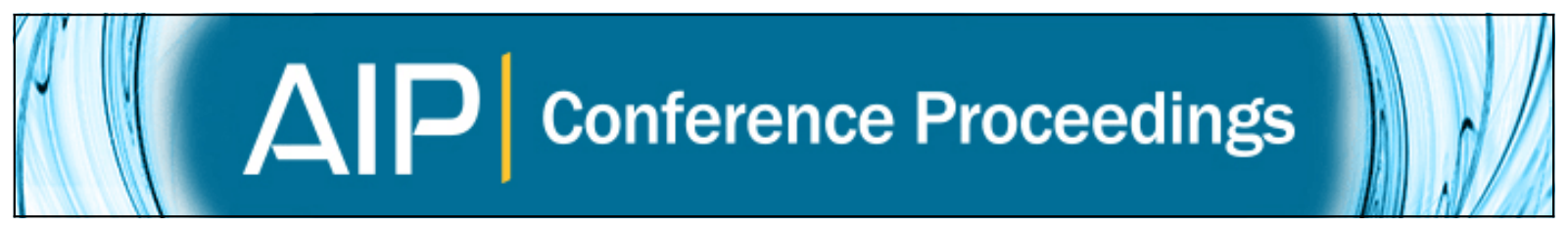

\title{
Fundamental Properties of Low-Mass Stars and Brown Dwarfs
}

Michael C. Liu, Keivan G. Stassun, France Allard, Cullen H. Blake, M. Bonnefoy, Ann Marie Cody, A. C. DayJones, Trent J. Dupuy, Adam Kraus, and Mercedes López-Morales

Citation: AIP Conference Proceedings 1094, 258 (2009); doi: 10.1063/1.3099100

View online: http://dx.doi.org/10.1063/1.3099100

View Table of Contents: http://scitation.aip.org/content/aip/proceeding/aipcp/1094?ver=pdfcov

Published by the AIP Publishing

\section{Articles you may be interested in}

Low-Mass Tertiary Companions to Spectroscopic Binaries: Common Proper Motion Survey for Wide Companions using 2MASS

AIP Conf. Proc. 1094, 824 (2009); 10.1063/1.3099243

The formation of brown dwarfs and low-mass stars by disc fragmentation

AIP Conf. Proc. 1094, 557 (2009); 10.1063/1.3099172

Determining the Physical Properties of Very-Low-Mass Stars and Brown Dwarfs in the Near-Infrared AIP Conf. Proc. 1094, 178 (2009); 10.1063/1.3099089

A Search for Pulsation in Very Low-mass Stars and Brown Dwarfs

AIP Conf. Proc. 948, 125 (2007); 10.1063/1.2818960

Formation of low mass stars and brown dwarfs

AIP Conf. Proc. 393, 217 (1997); 10.1063/1.52786 


\title{
Fundamental Properties of Low-Mass Stars and Brown Dwarfs
}

\author{
Michael C. Liu*, Keivan G. Stassun ${ }^{\dagger}$, France Allard ${ }^{* *}$, Cullen H. Blake ${ }^{\ddagger}$, \\ M. Bonnefoy ${ }^{\S}$, Ann Marie Cody ${ }^{\mathbb{I I}}$, A. C. Day-Jones", Trent J. Dupuy*, \\ Adam Kraus $^{\text {II }}$ and Mercedes López-Morales ${ }^{\dagger \dagger}$ \\ *Institute for Astronomy, University of Hawai 'i, 2680 Woodlawn Drive, Honolulu, HI 96822 \\ $\dagger$ Vanderbilt University, Physics \& Astronomy Department, Nashville, TN 37235 USA \\ ${ }^{* *}$ Centre de Recherche Astrophysique de Lyon, UMR 5574: CNRS, Université de Lyon, École \\ Normale Supérieure de Lyon, 46 allée d'Italie, 69364 Lyon Cedex 07 \\ ${ }^{\ddagger}$ Harvard-Smithsonian Center for Astrophysics, 60 Garden Street, Cambridge, MA, 02138 \\ ${ }^{\S}$ Laboratoire d'Astrophysique de Grenoble, BP 53, F-38041 GRENOBLE Cédex 9, France

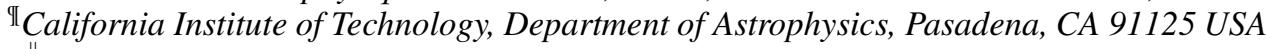 \\ "Centre for Astrophysics Research, University of Hertfordshire, Hatfield, AL10 9AB, UK \\ ${ }^{\dagger}$ Carnegie Institution of Washington, Dept. of Terrestrial Magnetism, Washington, DC 20015 USA
}

\begin{abstract}
Precise measurements of the fundamental properties of low-mass stars and brown dwarfs are key to understanding the physics underlying their formation and evolution. While there has been great progress over the last decade in studying the bulk spectrophotometric properties of low-mass objects, direct determination of their masses, radii, and temperatures have been very sparse. Thus, theoretical predictions of low-mass evolution and ultracool atmospheres remain to be rigorously tested. The situation is alarming given that such models are widely used, from the determination of the low-mass end of the initial mass function to the characterization of exoplanets.

An increasing number of mass, radius, and age determinations are placing critical constraints on the physics of low-mass objects. A wide variety of approaches are being pursued, including eclipsing binary studies, astrometric-spectroscopic orbital solutions, interferometry, and characterization of benchmark systems. In parallel, many more systems suitable for concerted study are now being found, thanks to new capabilities spanning both the very widest (all-sky surveys) and very narrowest (diffraction-limited adaptive optics) areas of the sky. This Cool Stars 15 splinter session highlighted the current successes and limitations of this rapidly growing area of precision astrophysics.
\end{abstract}

Keywords: Stars: fundamental parameters, low-mass, brown dwarfs, formation — Binary: general, close, eclipsing, visual - Instrumentation: adaptive optics, spectrographs

PACS: 97.21.+a, 95.85.Jq, 95.75.Fg, 95.75.Mn, 95.75.Qr, 97.10.Cv, 97.10.Ex, 97.80.Di, 97.90.+j, 97.20.Rp, 97.82.Fs

\section{VISUAL BINARIES}

About 100 ultracool (spectral type M6 or later) visual binaries are known, the product of several major high angular resolution imaging surveys conducted by HST and groundbased adaptive optics (AO) imaging (e.g., [1, 2, 3, 4, 5, 6, 7]). Most of these belong to the field population, near enough to Earth to be well-resolved and many have orbital periods amenable to dynamical mass determinations. A fundamental characteristic of field objects is that they span a range of (largely unknown) ages. This is a particularly important issue for brown dwarfs, which continually cool in time and thus follow a mass-luminosity-age relation. Despite this uncertainty, these objects can strongly test 


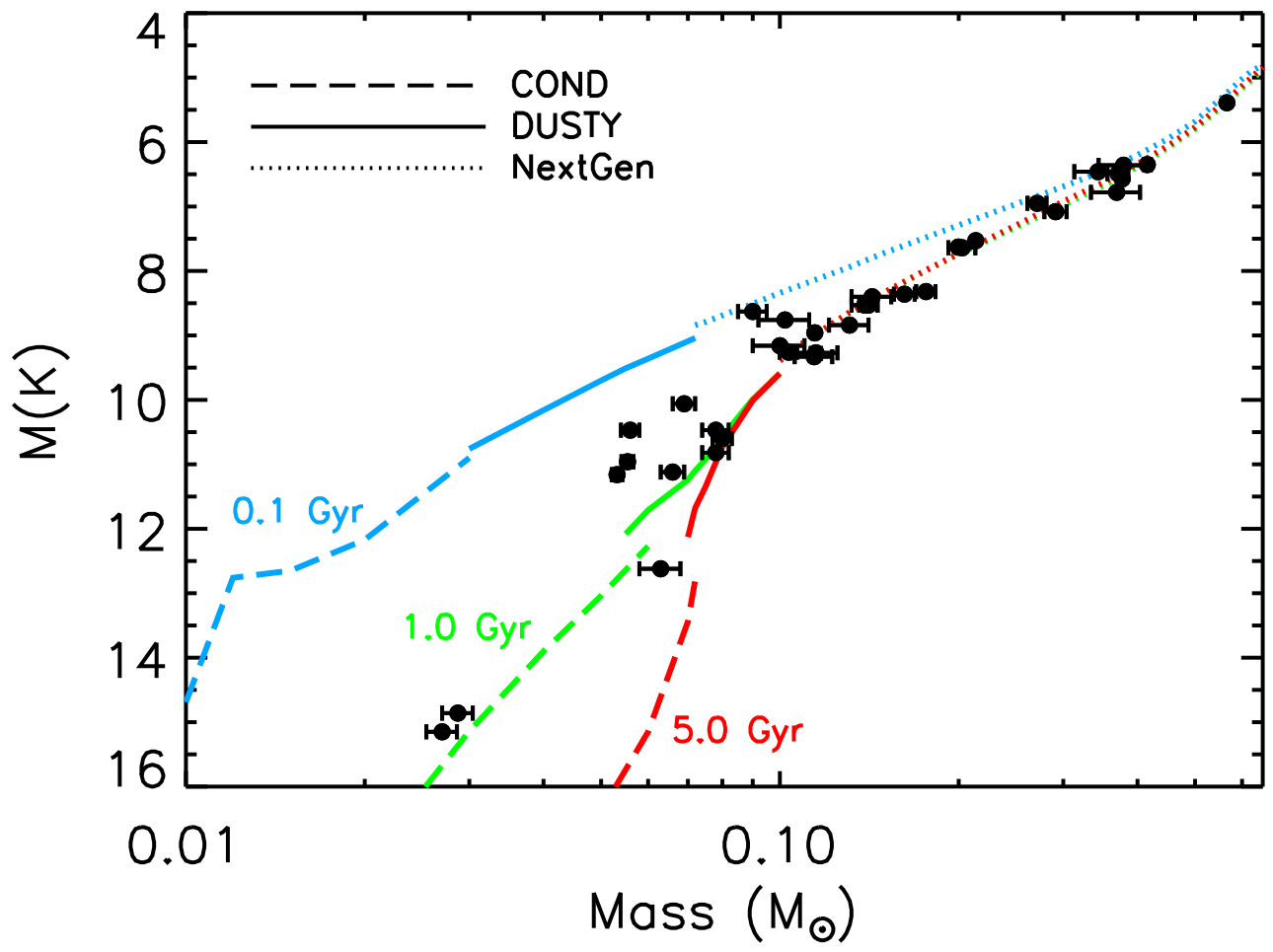

FIGURE 1. Absolute $K$-band magnitude as a function of dynamical mass for field M, L, and T dwarfs, based on data from [13, 14, 15, 16, 17, 18, 19, 10, 20]. For binaries where only the total mass is measured, the masses of the individual components are determined from the observed $K$-band flux ratio and evolutionary models to determine the mass ratio of the two components. The errors in the ordinate are comparable or smaller than the plotting symbol. Evolutionary models from [21, 22] are overplotted, with each class of models (NextGen, DUSTY or COND) plotted over the range of $T_{\text {eff }}$ appropriate for each model.

theoretical models when analyzed appropriately.

Accurate masses from visual binaries require high quality astrometry, radial velocities, and parallaxes (errors of $\sim 1$ mas, $\sim 1 \mathrm{~km} / \mathrm{s}, \sim 2 \%$, respectively). Also, to compare to models, independent determinations of $L_{b o l}$ to $\lesssim 10 \%$ (a more challenging measurement than appreciated at face value, e.g., [8]) and $T_{\text {eff }}$ are needed. Until this year, only three objects had dynamical masses that placed them unambiguously below the substellar limit: the M9 tertiary component of the hierarchical triple G1 569 [9, 10, 11] and both components of the young M6.5+M6.5 eclipsing binary 2MASS J05352184-0546085 in the Orion Nebula [12]. Since many of the first binary surveys were carried out nearly a decade ago, the next few years should see a rapid increase in the number of dynamical mass determinations, thereby extending the mass-magnitude relation by about a factor of 10 in mass and a factor of 100 in luminosity (Figure 1 .

Liu and collaborators have recently extended such measurements with the first dynamical mass for a binary T dwarf, the T5+T5.5 system 2MASS J1534-2952AB [16]. 
With a total mass of only of $0.056 \pm 0.003 M_{\odot}\left(59 \pm 3 M_{J u p}\right)$, this is the coolest and lowest mass binary with a dynamical mass to date, as well as the first field binary for which both components are confirmed to be substellar. The H-R diagram positions of the two components of 2MASS J1534-2952AB are discrepant with theoretical evolutionary tracks. While this could stem from large systematic errors in the luminosities ( $\sim 50 \%$ errors) and/or radii ( $\sim 20 \%$ errors) predicted by evolutionary models, the likely cause is that temperatures of mid-T dwarfs determined with model atmospheres are too warm by $\approx 100 \mathrm{~K}$. In fact, these model atmosphere uncertainties are the current limiting factor in testing theory using the H-R diagram, not the accuracy of the mass determinations. Morever, the prediction of different evolutionary models (e.g., Tucson and Lyon) are essentially indistinguishable on the H-R diagram.

These limitations imposed by atmospheric models can instead be circumvented, by using accurate mass and luminosity determinations in concert with evolutionary models to very precisely infer physical parameters for substellar binaries and assuming the systems are coeval. In the case of 2MASS J1534-2952AB, the formal uncertainties on the age $( \pm 0.1 \mathrm{Gyr})$, temperature $( \pm 17 \mathrm{~K})$ and surface gravity $( \pm 0.04$ dex $)$ allow for strong points of comparison with other data. For instance, this approach gives a relatively youthful age for the system of $0.79 \pm 0.09 \mathrm{Gyr}$, consistent with its low tangential velocity relative to other field T dwarfs. More generally, low-mass field binaries with dynamical mass determinations ("mass benchmarks") can serve as precise reference points for testing $T_{\text {eff }}$ and $\log (g)$ measurements from ultracool atmosphere models, as good as or even better than single brown dwarfs with age estimates ("age benchmarks"). In fact, given the plausible observational uncertainties, mass benchmarks are likely to provide stronger constraints (by a factor of $\approx 5$ ) on $\log (g)$ and $T_{\text {eff }}$ than age benchmarks, since dynamical masses can be determined far more accurately than ages for field stars.

Finally, the most stringent tests of theory can come from binaries with dynamical masses and independently determined ages, by being members of star clusters/groups and/or as companions to stars of known age. These systems represent the "gold standard" for testing models, but they are very rare. The splinter session presented new results for two objects:

- The Young Low-Mass Binary TWA 22AB: In 2004, Bonnefoy and collaborators resolved the TW Hydrae Association member TWA22 as a tight $(\sim 1.8$ AU) binary with the VLT/NACO instrument. Follow-up observations have monitored $80 \%$ of the binary orbit. Armed with the trigonometric parallax of the system, they find a total dynamical mass $\left(\mathrm{M}=220 \pm 21 M_{J u p}\right)$. Additional observations with the VLT/SINFONI AO-assisted integral field spectrometer have obtained medium resolution spectra $(R=1500-2000)$ of the primary and of the companion over the spectral range $1.0-2.5 \mu \mathrm{m}$. Spectral indices, equivalent widths and least-squares fitting were employed to compare the spectra to empirical spectral libraries of field and young dwarfs, yielding a $\mathrm{M} 6 \mathrm{~V} \pm 1$ spectral type for both components. Spectral templates were also used to estimate the temperatures and surface gravities of TWA22 A and B. Overall, the measured mass does not agree with 8-Myr evolutionary tracks. This could mean that TWA22 AB is either older than expected (and perhaps not a member of TWA) or that models under-predict masses of young objects.

- The Field Substellar Benchmark Binary HD 130948BC: Dupuy and collaborators 

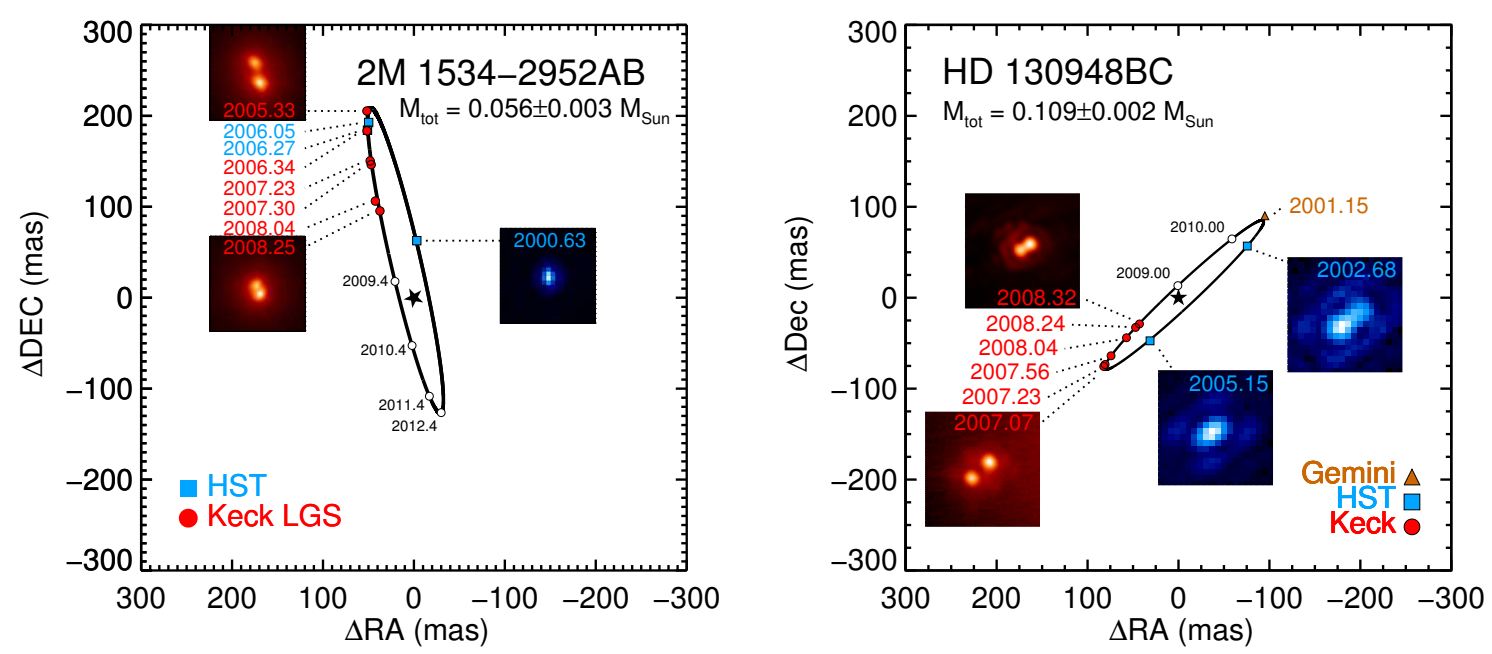

FIGURE 2. Recent orbit and dynamical mass determinations for field brown dwarf binaries. The insets show imaging data at selected epochs and are $1^{\prime \prime}$ on a side. Left: Orbit for the binary T5.0+T5.5 dwarf 2MASS J1534-2952AB, based on high angular resolution monitoring using HST and Keck laser guide star adaptive optics [16]. This is the coolest and lowest mass visual binary to date with a direct mass determination. Right: Orbit for the binary L4+L4 dwarf HD 130948BC, based on HST and natural guide star imaging from Keck and Gemini [17]. This system also has an independent age determination of $0.79_{-0.15}^{+0.22} \mathrm{Gyr}$, based on the rotation and activity properties of its G2V primary star, making it a thus far unique benchmark system for testing theoretical models.

have been using the Keck AO system to monitor a large sample of ultracool field binaries to enable a much better assessment of substellar theoretical models by obtaining many more dynamical masses. They presented Keck AO imaging of the L4+L4 binary HD 130948BC along with archival HST and Gemini-North observations, which together span $\approx 70 \%$ of the binary's orbital period [17]. From the relative orbit, they determine a total dynamical mass of $0.109 \pm 0.002 \mathrm{M}_{\odot}\left(114 \pm 2 \mathrm{M}_{\mathrm{Jup}}\right)$. The flux ratio of HD 130948BC is near unity, so both components are unambiguously substellar for any plausible mass ratio. In addition, an independent constraint on the age of the system is available from the primary HD 130948A $(\mathrm{G} 2 \mathrm{~V},[\mathrm{M} / \mathrm{H}]=0.0)$. The ensemble of available indicators suggests an age comparable to the Hyades, with the most precise age being $0.79_{-0.15}^{+0.22}$ Gyr based on gyrochronology.

As a result, HD 130948BC is now a unique benchmark among field L and T dwarfs: it is the only system with a well-determined mass, luminosity, and age. Thus, the luminosity evolution of brown dwarfs predicted by theoretical models is fully constrained by observations for the first time, and the result is that the models disagree with the data: (1) Both components of HD 130948BC appear to be overluminous by a factor of $\approx 2-3 \times$ compared to evolutionary models. The age of the system would have to be notably younger than the gyro age to ameliorate the luminosity disagreement. (2) Effective temperatures derived from evolutionary models for HD 130948B and C are inconsistent with temperatures determined from spectral synthesis for objects of similar spectral 
type. Overall, regardless of the adopted system age, evolutionary and atmospheric models give inconsistent results, which indicates systematic errors in at least one class of models, possibly both. The masses of HD 130948BC happen to be very near the theoretical mass limit for lithium burning, such that the Lyon and Tucson models give drastically different predictions for the lithium depletion that has occured in each component. Thus, measuring the differential lithium depletion between $\mathrm{B}$ and $\mathrm{C}$ will provide a uniquely discriminating test of theoretical models.

The potential underestimate of luminosities by evolutionary models would have wideranging implications since they are widely used for characterizing low-mass stars, brown dwarfs, and planets. Therefore, a more refined age estimate for HD 130948A and measurements for more such mass+age benchmarks are critically needed to determine the magnitude of the luminosity discrepancy more precisely.

\section{ECLIPSING BINARIES}

Eclipsing binary (EB) stars generally offer the most accurate means for directly measuring stellar masses and radii. Of course, EBs are rare, and thus the mass-radius relation of $\mathrm{K}$ and $\mathrm{M}$ dwarfs has historically been poorly constrained.

The situation has improved recently with the discovery of several new detached EBs, particularly at very low masses and at young ages, as described in presentations by Stassun, López-Morales, and Kraus. For example, in the last few years the number of young $(<30 \mathrm{Myr})$, low-mass $\left(M<2 \mathrm{M}_{\odot}\right)$ EBs has increased to 16 (see [23]). Highlights from this recent work include: (1) The first EB of equal-mass ("identical twin") stars that exhibit striking differences in temperature and luminosity, suggesting that non-coevality of $\sim 30 \%$ is possible in young binaries [24], and (2) the first EB system of two brown dwarfs [12, 25] in which strong magnetic activity is likely responsible for the surprising reversal of temperatures in the system (the higher mass brown dwarf is the cooler of the pair). Indeed, there is now growing evidence that magnetic activity may be affecting the structure, and thus the basic mass-luminosity relationship, of young, low-mass stars.

Among field stars, the results from EBs combined with stellar radii of single $\mathrm{K}$ and $\mathrm{M}$ dwarfs derived via interferometry (in which case the stellar masses are derived from models) currently add up to over forty radius measurements that can test stellar models. A graphical summary is shown in Fig. 2, which plots all the available mass and radius

measurements for stars with $M<1 M_{\odot}$ versus the best fitting model from [22]. The figure clearly illustrates that the radii of many of the stars are significantly larger than predicted by the models for stars with $M>0.35 M_{\odot}$, and there also is significant scatter in the stellar radii. For stars with $M<0.35 M_{\odot}$, which coincides with stars becoming fully convective, models and observations seem to agree (but see also below).

The most plausible explanations for these trends are the magnetic activity in the atmospheres of these stars, or their metallicity (equation of state effects cannot currently be tested observationally). Magnetic activity is clearly affecting the radii of the stars, at least in the case of the most active components of binaries (see Fig. 2 of [26]). In the case of single stars, this radius-magnetic activity correlation is not as clear. However, we need to address that this conclusion might suffer from an observational bias, as all the stars with $M<0.35 M_{\odot}$ in the sample happen to have low activity levels. Metallicity 

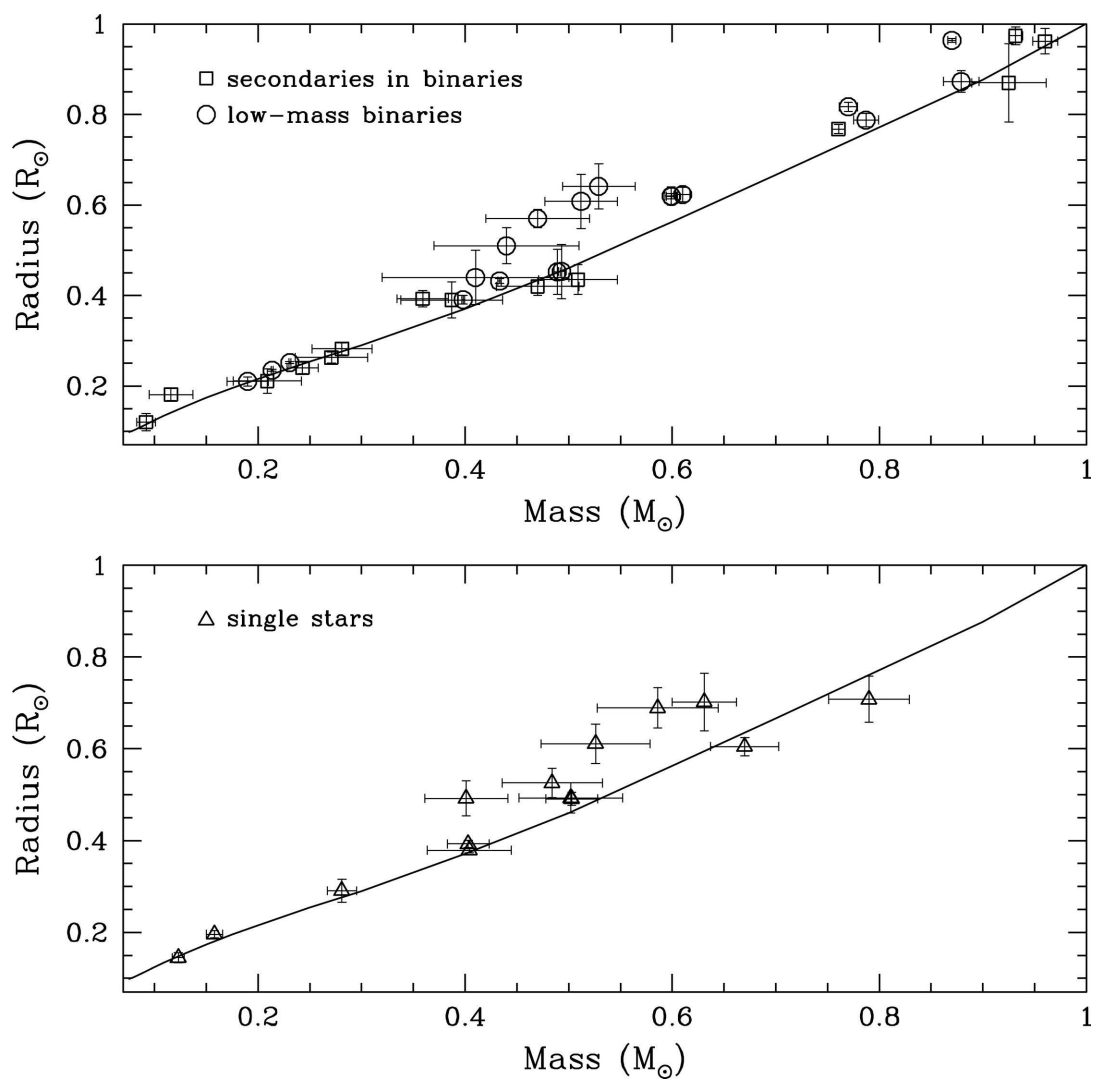

FIGURE 3. Current observational mass-radius relation for stars below $1 \mathrm{M}_{\odot}$. Top, all the data from low-mass secondaries to eclipsing binaries with primaries more massive than $1 \mathrm{M}_{\odot}$ (squares) and the components of eclipsing binaries below $1 \mathrm{M}_{\odot}$ (circles); bottom, all the measurements from single stars. The solid line in each panel represents the theoretical isochrone model from [22], for an age of $1 \mathrm{Gyr}, \mathrm{Z}$ $=0.02$, and mixing length $\alpha=1.0$ (standard model). Figure reproduced from [26] by permission of the AAS.

seems to also be having some effect in the radii of $\mathrm{K}$ and $\mathrm{M}$ dwarfs stars, with more metal-rich objects apparently showing larger radii (see Fig. 4 of [26]). However, the still weakly determined metallicity scale of low-mass stars, and the scatter in the data, might prove this last correlation spurious.

More radius measurements of low-metallicity low-mass stars are necesary. To that end, work is ongoing to increase the sample of low-mass EBs further. M dwarfs in particular are ubiquitous in the solar neighborhood, but their fundamental properties are not as well understood as those of their more massive brethren. Only $\sim 12 \mathrm{M}$-dwarf EBs have been identified to date since low-mass stars are intrinsically faint and shallow variability surveys have only encompassed a very limited survey volume. Kraus and collaborators are conducting a program to identify and characterize new M dwarf EBs from a deep field variability survey (the 1st MOTESS-GNAT survey; [27]). Thus far, they have identified $\sim 25$ new M dwarf EBs with spectral types as late as M4; this sample triples the number of known systems. They have obtained radial velocity curves for 18 of these systems with Palomar, Keck, and Hobby-Eberly, more than doubling the number of precise mass measurements for $\mathrm{M}$ dwarf EBs, and now they are pursuing an ongoing 
program to obtain multicolor eclipse light curves in order to measure the component radii for each system. When complete, this survey will allow for the first systematic investigation of the fundamental properties of very low-mass stars.

\section{ATMOSPHERIC MODELS}

The atmospheres of very low-mass stars and brown dwarfs are governed by the formation of molecules and dust grains in a relatively high-temperature, high-gravity environment compared to laboratory experiments. Over the past decades, it has been shown that these opacities cover all wavelengths of the emerging spectrum, to the point of locking the peak of the SED around $1.2 \mu \mathrm{m}$ as $T_{\text {eff }}$ decreases for metal-rich compositions. The effect of reducing metallicity would therefore be to recover the reddening of the SED with decreasing $T_{\text {eff }}$. However, double metals deplete more rapidly and thus densitysensitive features such as hydride absorption bands $(\mathrm{MgH}$ and $\mathrm{CaH})$ and the very important near-infrared collision-induced $\mathrm{H}_{2}$ absorption are revealed and shape the SED in enhanced-density atmospheres. The effects of changing surface gravity are relatively more subtle and consist of atmospheric density changes at constant composition, which can be compensated by a $T_{\text {eff }}$ change in $\mathrm{M}$ dwarfs. There is therefore a degeneracy of solutions in determining the gravity and $T_{\text {eff }}$, and therefore age and mass, of very low-mass stars and brown dwarfs.

Atomic line profile analysis is complicated by the uncertain molecular pseudocontinuum background (oscillator strengths are often inaccurate or missing). In addition, for T dwarfs an additional complication is the lack of this pseudo-continuum background, causing the line wings of atomic lines to shape the SED more than $2000 \AA$ from the line center, i.e. beyond the validity of classical assumptions for the line profile modeling (Lorentz profile with van der Waals collisional broadening).

Allard and collaborators have developed an online tool (web simulator) for the determination of parameters based on observed colors by chi-square fitting and also generating isochrones on any model grid, filter set, and reddening. Among the challenges for modeling these atmospheres and therefore improving the determination of their parameters will be improvement of (1) the molecular opacities and line broadening of alkali metals (and molecules), and (2) the modeling of clouds and non-equilibrium chemistry, which both depend on understanding of mixing induced by convection into the line formation layers of these atmospheres.

\section{OTHER RECENT DEVELOPMENTS}

An L Dwarf Radial Velocity Survey: Precise radial velocities (RVs) of very low-mass stars and brown dwarfs can provide a wealth of information about the fundamental physical properties of these objects. Measurements of projected rotational velocities and systemic velocities, when coupled with proper motions, provide insight into the dynamical history of this population. In addition, multi-epoch RV measurements can be used to search for single- and double-lined binaries. Binaries provide an excellent opportunity to directly measure the fundamental physical properties of stars and brown 
dwarfs and to compare these measurements to theoretical models.

Blake and collaborators have developed a technique for obtaining precise RVs of low-mass stars and brown dwarfs in the near infrared and are conducting a magnitudelimited survey of L dwarfs with the NIRSPEC spectrograph on the Keck telescope. The sample consists of $75 \mathrm{~L}$ dwarfs with observations spanning up to four years. With a typical RV precision of $200 \mathrm{~m} \mathrm{~s}^{-1}$, they are very sensitive to low-mass binaries with orbital separations smaller than those probed by direct imaging techniques. They have discovered one new single-lined L dwarf binary, 2MASS 0320-04, likely comprised of a late-M and a T dwarf ([28]; see also [29]). A more detailed analysis of the RV data for this system may result in the detection of the spectral lines of the fainter component. This would provide a direct measurement of both the mass and luminosity ratios of the binary components, allowing us to directly test theoretical models of brown dwarfs.

Searching for benchmark brown dwarfs as members of binary systems: For the majority of known brown dwarfs, properties such as gravity and metallicity remain uncertain, and it is not yet fully understood how factors these affect their spectra or change over time. The complex nature of ultracool and brown dwarf atmospheres leaves models incomplete or with large uncertainties on their values. What is vitally needed are benchmark objects, where properties can be determined independently.

Day-Jones and collaborators are currently undertaking a search to find such benchmarks as members of binary systems containing an age-calibratable primary. White dwarf or subgiant primaries can provide accurate ages, distances and, for subgiants, metallicity constraints, which can then be applied (by association) to the brown dwarf companions. They have searched for widely separated ( 20,000 AU) ultracool+white dwarf binaries in 2MASS and SuperCOSMOS in the southern hemisphere and have to date discovered one ultracool+white dwarf binary system [30], which is the widest separated M9 + WD binary known to date and has an age constraint of $>1.94$ Gyrs. To find similar systems, they have also mined the latest releases of SDSS (DR6) and UKIDSS Large-Area Survey (DR3) and carried out a pilot imaging survey in the south; these have yielded a good number of candidates that are currently being followed up.

Searching for Pulsation in Brown Dwarfs and Very Low Mass Stars: Censuses of young ( $\sim 1-10 \mathrm{Myr})$ clusters over the past decade have revealed substantial numbers of very low mass stars and brown dwarfs. In order to place more constraints on the physical properties of young brown dwarfs, Cody and collaborators have begun a photometric campaign that aims to uncover a pulsational instability on hour timescales in those objects that are still burning deuterium [31]. The identification of pulsations would provide a new probe of brown dwarf interiors through the physics of seismology. The campaign is ongoing, with completed or planned observations of some 80 brown dwarfs and very low mass stars in five young star clusters, using telescopes at the Palomar and CTIO. Preliminary results in the IC 348 and $\sigma$ Orionis clusters include several brown dwarfs displaying variability with periods of a few hours at the limit of detectability. High-resolution spectroscopic followup is underway to determine whether rapid rotational modulation of magnetic spots can explain the light curves, or if the variability can indeed be attributed to a new class of pulsation. 


\section{REFERENCES}

1. I. N. Reid, J. E. Gizis, J. D. Kirkpatrick, and D. W. Koerner, AJ 121, 489-502 (2001).

2. H. Bouy, W. Brandner, E. L. Martín, X. Delfosse, F. Allard, and G. Basri, AJ 126, 1526-1554 (2003).

3. A. J. Burgasser, J. D. Kirkpatrick, I. N. Reid, M. E. Brown, C. L. Miskey, and J. E. Gizis, ApJ 586, 512-526 (2003).

4. L. M. Close, N. Siegler, M. Freed, and B. Biller, ApJ 587, 407-422 (2003).

5. M. C. Liu, and S. K. Leggett, $A p J$ 634, 616 (2005).

6. M. C. Liu, S. K. Leggett, D. A. Golimowski, K. Chiu, X. Fan, T. R. Geballe, D. P. Schneider, and J. Brinkmann, ApJ 647, 1393 (2006).

7. I. N. Reid, K. L. Cruz, A. J. Burgasser, and M. C. Liu, AJ 135, 580-587 (2008).

8. D. A. Golimowski, et al., $A J$ 127, 3516-3536 (2004).

9. B. F. Lane, M. R. Zapatero Osorio, M. C. Britton, E. L. Martín, and S. R. Kulkarni, ApJ 560, 390-399 (2001).

10. M. R. Zapatero Osorio, B. F. Lane, Y. Pavlenko, E. L. Martín, M. Britton, and S. R. Kulkarni, ApJ 615, 958-971 (2004).

11. M. Simon, C. Bender, and L. Prato, ApJ 644, 1183-1192 (2006).

12. K. G. Stassun, R. D. Mathieu, and J. A. Valenti, Nature 440, 311-314 (2006).

13. X. Delfosse, T. Forveille, D. Ségransan, J.-L. Beuzit, S. Udry, C. Perrier, and M. Mayor, $A \& A$ 364, 217-224 (2000).

14. F. Martinache, J. P. Lloyd, M. J. Ireland, R. S. Yamada, and P. G. Tuthill, ApJ 661, 496-501 (2007).

15. M. J. Ireland, A. Kraus, F. Martinache, J. P. Lloyd, and P. G. Tuthill, ApJ 678, 463-471 (2008).

16. M. C. Liu, T. J. Dupuy, and M. J. Ireland, $A p J$ (2008), in press (astro-ph/0807.0238).

17. T. J. Dupuy, M. C. Liu, and M. J. Ireland, $A p J$ (2008), in press (astro-ph/0807.2450).

18. H. Bouy, et al., $A \& A$ 423, 341-352 (2004).

19. A. Seifahrt, T. Röll, R. Neuhäuser, A. Reiners, F. Kerber, H. U. Käufl, R. Siebenmorgen, and A. Smette, $A \& A$ 484, 429-434 (2008).

20. L. M. Close, N. Thatte, E. L. Nielsen, R. Abuter, F. Clarke, and M. Tecza, ApJ 665, 736-743 (2007).

21. I. Baraffe, G. Chabrier, T. S. Barman, F. Allard, and P. H. Hauschildt, $A \& A$ 402, 701-712 (2003).

22. I. Baraffe, G. Chabrier, F. Allard, and P. H. Hauschildt, $A \& A$ 337, 403-412 (1998).

23. R. D. Mathieu, I. Baraffe, M. Simon, K. G. Stassun, and R. White, "Dynamical Mass Measurements of Pre-Main-Sequence Stars: Fundamental Tests of the Physics of Young Stars," in Protostars and Planets V, edited by B. Reipurth, D. Jewitt, and K. Keil, 2007, pp. 411-425.

24. K. G. Stassun, R. D. Mathieu, P. A. Cargile, A. N. Aarnio, E. Stempels, and A. Geller, Nature 453, 1079-1082 (2008).

25. K. G. Stassun, R. D. Mathieu, and J. A. Valenti, ApJ 664, 1154-1166 (2007).

26. M. López-Morales, ApJ 660, 732-739 (2007).

27. A. L. Kraus, E. R. Craine, M. S. Giampapa, W. W. G. Scharlach, and R. A. Tucker, AJ 134, 14881502 (2007).

28. C. H. Blake, D. Charbonneau, R. J. White, G. Torres, M. S. Marley, and D. Saumon, ApJ 678, L125-L128 (2008).

29. A. J. Burgasser, M. C. Liu, M. J. Ireland, K. L. Cruz, and T. J. Dupuy, ApJ 681, 579-593 (2008).

30. A. C. Day-Jones, D. J. Pinfield, R. Napiwotzki, B. Burningham, J. S. Jenkins, H. R. A. Jones, S. L. Folkes, D. J. Weights, and J. R. A. Clarke, MNRAS 388, 838-848 (2008).

31. F. Palla, and I. Baraffe, $A \& A$ 432, L57-L60 (2005). 